\title{
SEM and EBSD Investigations of High-Chromium Cast Irons
}

\author{
Brook Hinckley ${ }^{*}$, Kevin F. Dolman ${ }^{*}$, Richard Wuhrer ${ }^{* *}$, Abhi Ray ${ }^{* * *}$, Wing Yeung ${ }^{* * *}$ \\ *Weir Minerals Australia Ltd, Locked Bag 51, Artarmon NSW 1570, Australia \\ ** Microstructural Analysis Unit and ${ }^{* * *}$ Department of Chemistry, Materials and Forensic \\ Science, University of Technology, PO Box 123, Broadway, Sydney, NSW 2007, Australia
}

\section{Introduction}

High-chromium white cast irons are alloys that are based on the iron-chromium-carbon system and are classified in ISO 21988-2006 as abrasion-resistant cast irons that contain greater than 11\% chromium. High-chromium white irons are primarily used as castings and hard-facing weld deposits in equipment requiring resistance to abrasive and erosive wear under moderate impactloading conditions. These materials are used in the mining and chemical processing plants in applications such as grinding mills, slurry pumps, chute liners and pipes.

The microstructures of high-chromium white irons contain a substantial proportion of chromiumrich carbides that are harder than silica sand, which is a commonly encountered wear medium in mineral processing plants. High-chromium white irons are generally heat treated to develop maximum hardness via the precipitation of secondary carbides in the ferrous matrix during ageing at elevated temperatures and destabilisation of austenite, which subsequently transforms to martensite on cooling to room temperature.

High chromium white cast irons undergo several solidification reactions and a number of different solid state transformation reactions on cooling to room temperature and reheating to elevated temperatures below the solidus. Consequently, a number of different phases form in highchromium white irons that influence the mechanical properties and service life of the material. The following transformation reactions have been observed to occur in high chromium white cast irons:

- Primary austenite in hypoeutectic alloys.

- Primary $\mathrm{M}_{7} \mathrm{C}_{3}$ carbides in hypereutectic alloys.

- Eutectic carbide and austenite in hypoeutectic and hypereutectic alloys.

- Eutectic ferrite in hypoeutectic alloys.

- Peritectic carbides in hypereutectic alloys.

- Secondary carbide precipitation during slow cooling or upon reheating.

- Lamellar pearlite, and decomposition of austenite to martensite.

- Tempered martensite to ferrite, and of ferrite to austenite.

- Dissolution of pearlite to austenite and dissolution of secondary carbides.

In this present study, microstructures of a several high-chromium white iron alloys were examined in the as-cast, solution treated and hardened conditions. Scanning electron microscopy (SEM) and electron back-scattered diffraction (EBSD) were found to be useful for identifying various transformation reaction products for heat treated white irons. 


\section{Results and Discussion}

High-chromium white iron test plates, 180 x 50 x $13 \mathrm{~mm}$ in size, were cast from a $25 \mathrm{~kg}$ induction melting furnace into sand moulds. Specimens for SEM and EBSD studies were prepared using standard metallographic techniques followed by electropolishing at $37-42 \mathrm{~V}$ in a solution of $5 \%$ perchloric acid in ethanol at temperatures between $10^{\circ} \mathrm{C}$ and ambient temperature. SEM and EBSD specimens were specifically produced in the unmounted condition in order to maximise electrical conduction, thereby minimising charging and drift effects during exposure to the electron beam. An HKL Technology EBSD system attached to a Zeiss Supra 55VP FEGSEM was operated at $20 \mathrm{kV}$ accelerating voltage, $20 \mathrm{~mm}$ working distance, $120 \mu \mathrm{m}$ aperture and high current mode for EBSD analysis.

In the as-cast condition, the microstructure consisted of primary and eutectic $\mathrm{M}_{7} \mathrm{C}_{3}$ carbides in a wholly pearlitic matrix, Figure 1a. In the solution treated and quenched condition, the ferrous matrix was wholly austenitic and characteristic annealing twins were evident, Figure $1 \mathrm{~b}$. The precipitation-hardened microstructure consisted of primary and eutectic $\mathrm{M}_{7} \mathrm{C}_{3}$ carbides in a ferrous matrix of martensite and a small amount of retained austenite, Figure 1c.

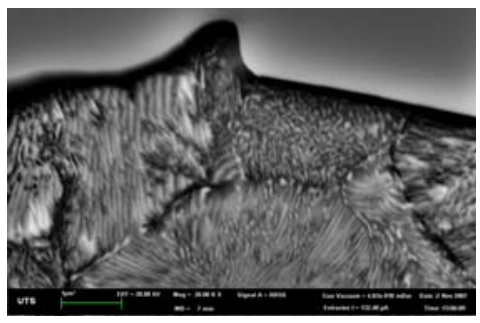

a.

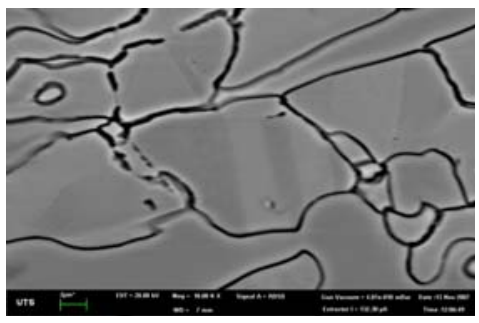

b.

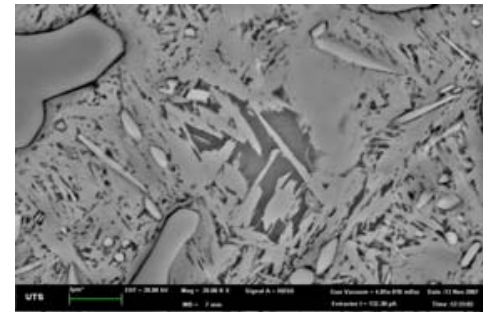

c.

Figure 1: Microstructures of the high-chromium cast iron in various conditions;

(a) in as-cast condition, (b) after solution treatment at $1200^{\circ} \mathrm{C}$

(c) after age-hardening treatment at $950^{\circ} \mathrm{C}$.

EBSD studies confirmed the crystal structures of the austenite (Space Group 225) and martensite (SG229) in the ferrous matrix, Figures $2 \& 3$. The primary and eutectic carbides were hexagonal (SG186) although they were also similar to the orthorhombic structure (SG62).

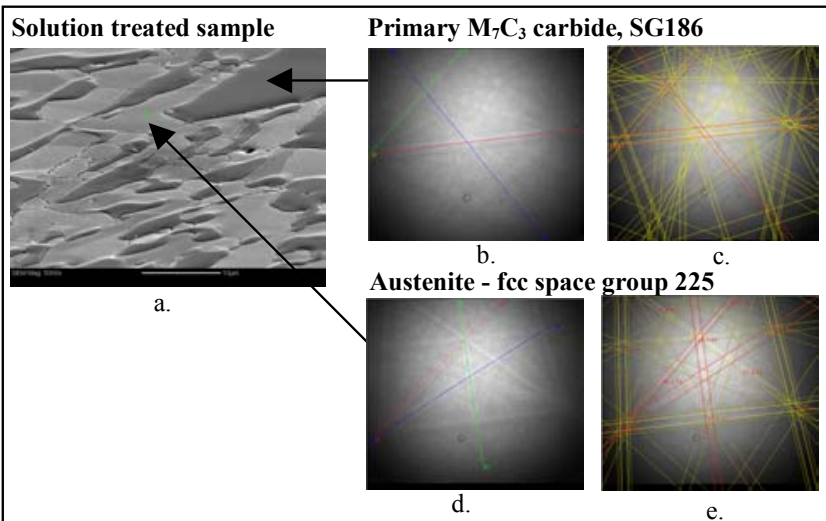

Figure 2: Solution treated sample; (a) microstructure, (b) EBSD patterns and (c) indexed EBSD result for primary carbide, (d) EBSD pattern and (e) indexed EBSD result for the austenite phase.

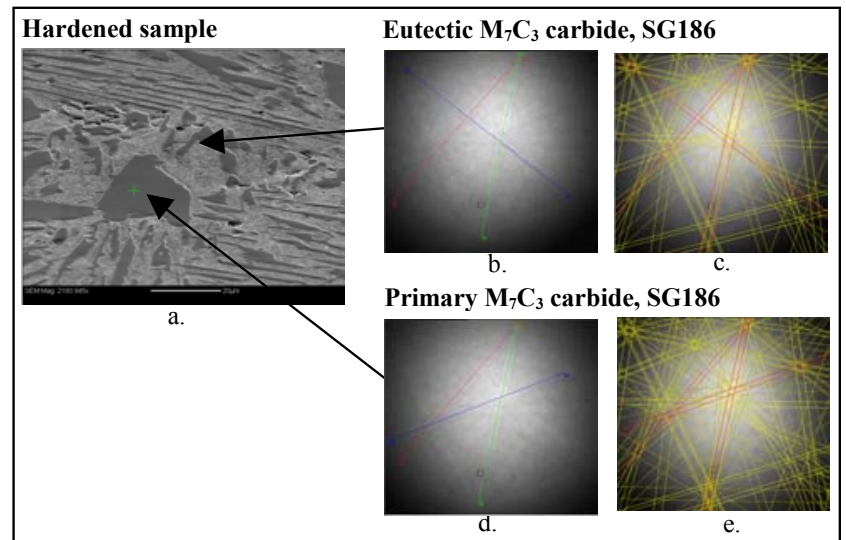

Figure 3: Age-hardened sample; (a) microstructure, (b) EBSD patterns and (c) indexed EBSD result for eutectic carbide, (d) EBSD pattern and (e) indexed EBSD result for the primary carbide. 